\title{
A Study of Some Properties of Bottomonium
}

\author{
A. M. Yasser'1, G. S. Hassan' ${ }^{2}$, T. A. Nahool ${ }^{1}$ \\ ${ }^{1}$ Physics Department, Faculty of Science, South Valley University, Qena, Egypt \\ ${ }^{2}$ Physics Department, Faculty of Science, Assiut University, Asyut, Egypt \\ Email: Yasser.mostafa@sci.svu.edu.eg, tarek.abdelwahab@sci.svu.edu.eg
}

Received 5 September 2014; revised 1 October 2014; accepted 25 October 2014

Copyright (C 2014 by authors and Scientific Research Publishing Inc.

This work is licensed under the Creative Commons Attribution International License (CC BY).

http://creativecommons.org/licenses/by/4.0/

cc) (i) Open Access

\begin{abstract}
We apply matrix Numerov's method to obtain the radial wave functions; from these wave functions we calculate the root mean square radius $r_{m s}$ and $\beta$ coefficients of bottomonium $b \bar{b}$. The obtained results have implications for decay constants, decay widths and differential cross sections of heavy mesons.
\end{abstract}

\section{Keywords}

Matrix Numerov's Method, Wave Functions, $\beta$ Coefficient, Root Mean Square Radius, Bottomonium

\section{Introduction}

Quarkonium in particle physics refers to meson whose constituents are a quark and its own antiquark. The famous quarkonium system is charmonium and bottomonium. Bottomonium $b \bar{b}$ meson has discovered recently with the ATLAS detector at the Large Hadron Collider (LHC) [1]. Bottomonium family is the set of particles that contain both a bottom quark and an anti-bottom quark but are bound together with different energies. A number of botommonium properties are well described by the quark model [2]-[8] where mesons have quantum numbers $J=L \oplus S, P=(-1)^{L+1}$ and $C=(-1)^{L+S} ; L$ and $S$ are the quantum numbers for the quark-antiquark orbital angular momentum and their net spin angular momentum respectively [9]. The main aim of our work is to study the spectra of heavy mesons and the corresponding wave functions. Bottomonium $b \bar{b}$ spectra, as an example of heavy meson, are investigated by using matrix Numerov's method [10] [11] via non-relativistic potential model [12]-[14]. However, a vast majority of numerical methods have been used to solve the Schrödinger equation (SE) numerically, for instance, Runge-Kutta method [15], Shooting method [16], Numerov's method [17], four-step exponentially fitted method [18] and the factorization method [19]. But, here we show that the matrix Numerov's algorithm is a more efficient and fast one to achieve our goal; we hope this approximation gives the reliability features of heavy meson investigation. Moreover, the heavy-meson wave functions deter- 
mined in this work can be employed to make predictions of other properties. On the other hand, the main motivation is to calculate the root mean square radius $r_{m s}$ of different states for bottomonium and the numerical values of $\beta$ coefficient, which can be used to calculate the decay widths [20], and differential cross sections [21] for quarkonium states. Besides, an additional aim of our work is to investigate the mass-radius dependence for states of bottomonium. The remainder of this paper is organized as follows. In Section 2, we present some characteristics properties of bottomonium mesons which in turn depend on the potential model. In Section 3, we present our main problem and its analytic solution. In Section 4, results and discussion are given. Finally in the last section, we summarize our main results and conclusions.

\section{Characteristics of Bottomonium Mesons}

\subsection{The Potential Model of Bottomonium Mesons}

One of the most successful ways of describing the quarkonium system is to solve the non-relativistic Schrödinger equation for these quark-anti quark states with an appropriate potential model. In a non-relativistic constituent quark model, one ignores the dynamical effects of gluon fields on the hadrons structure and properties. Quarks are considered as non-relativistic objects interacting via an instantaneous adiabatic potential provided by gluons, and the non relativistic description with the Schrödinger equation gives acceptable results.

Thus, the potential model used here [22] [23] is written as:

$$
V_{N}(r)=\frac{l(l+1)}{2 \mu r^{2}}-\frac{4 \alpha_{s}}{3}+b r+\frac{32 \pi \alpha_{s}}{9 m_{b}^{2}} \delta(r) S_{b} S_{\bar{b}}+\frac{1}{m_{b}^{2}}\left[\left(\frac{2 \alpha_{s}}{r^{3}}-\frac{b}{2 r}\right) \boldsymbol{L} \cdot \boldsymbol{S}+\frac{4 \alpha_{s}}{r^{3}} T\right]
$$

where $S_{b} S_{\bar{b}}=\frac{s(s+1)}{2}-\frac{3}{4}, \mu$ is the reduced mass of the quark and anti-quark, $m_{b}$ is the mass of the bottom quark, and $S$ is the total spin quantum number of the meson. For the $b \bar{b}$ mesons, the parameters $\alpha_{s}, b, \sigma$, and $m_{b}$ are taken to be $0.4036,0.1624 \mathrm{GeV}, 2.4948 \mathrm{GeV}$ and $4.8097 \mathrm{GeV}$ respectively [24]. $T$ is the tensor operator and the spin-orbit operator is diagonal in a $|J, L, S\rangle$ basis [9], with the matrix elements.

$$
\langle\boldsymbol{L} \cdot \boldsymbol{S}\rangle=\frac{[J(J+1)-(L(L+1)-S(S+1))]}{2}
$$

\subsection{Wave Functions of Bottomonium Mesons}

Bottomonium mesons can be described by the wave function of the bound quark-antiquark state which satisfies the SE by using the potential given in Equation (1). Radial Schrödinger equation, $\psi(r)=r R(r)$, is written (in natural units) as:

$$
\psi(r)=2 \mu(E-V(r)) \psi(r)=0
$$

where $R(r)$ is the radial wave function, $r$ is the inter quark distance, $E$ is the sum of kinetic and potential of quark-antiquark system, and $V(r)$ and $\mu$ are defined above through Equation (1). The matrix Numerove's method is used to solve Equation (1) to get spectra of bottomonium, the detailed of this method could be found in Ref. [11]. In the following sections, we employ that method to obtain the wave functions of bottomonium.

\section{Basic Properties of Bottomonium Meson}

\subsection{Bottomonium Root Mean Square Radius $r_{m s}$}

Define Bottomonium root mean square radius $r_{m s}$ is one of basic properties of bottomonium. If the distance between the quark and anti-quark in bottomonium is $r$ fm it may be regarded that bottomonium has radius $r / 2 \mathrm{fm}$ where $r$ is the distance from the point quark to anti-quark. $r_{m s}$ can be derived from the meson wave function and may be written as [25].

$$
i r_{m s}^{2}=\int_{0}^{\infty}\left\{\psi^{2}(r) r^{2} \mathrm{~d} r\right\}
$$




\section{2. $\beta$ Coefficient}

The meson wave function is characterized by a momentum width parameter $\beta$ that is related to the root mean square quark-antiquark separation $r_{m s}$ of the meson by [26].

$$
\beta=\sqrt{2(n-1)+(L)+\frac{3}{2}} \frac{1}{r_{m s}}
$$

where $n$ is the principal quantum number and $L$ is the sub-atomic energy level number. The parameter $\beta$ is typically taken as a parameter of the model. However, since we are seeking for describing the decay of heavy quark states, it is preferable to reproduce $\beta$ coefficient of the quark model states. These values of $\beta$ are obtained for the first time. So, we suggest using it to calculate the decay width of heavy quarkonium states.

\section{Results and Discussion}

A non-relativistic potential model is used to study some properties of bottomonium meson by using the matrix Numerov's method. The eigenvalues and the corresponding wave functions are found by using the same method. Then we normalized the wave functions and found the root mean square radius of bottomonium mesons by using Equation (3). Moreover, we can obtain computational values of $\beta$ coefficient by using Equation (4). The normalized radial wave functions for bottomonium mesons are graphically represented in Figures 1-3 respectively. For bottomonium mesons, our calculated masses and root mean square radius are reported in Table 1 in case of S, P and D States respectively. We observe that our results are in good agreement with the experiment [27] and existing theoretically predicted values [24], which shows the validity of the used method. Some of our calculated root mean square radii are found to be in good agreement with the published one of ref. [28]. The $\beta$ values could be used to calculate the decay constants [29], decay widths [29], and differential cross sections [30] for quarkonium states with high accuracy as we used complicated potential model. The predictions about these quantities are also reported in Table 1 for bottomonium S, P, and D States respectively. Finally, we investigated the mass-radius dependence for states of bottomonium. We confirmed a leading linear relation between masses

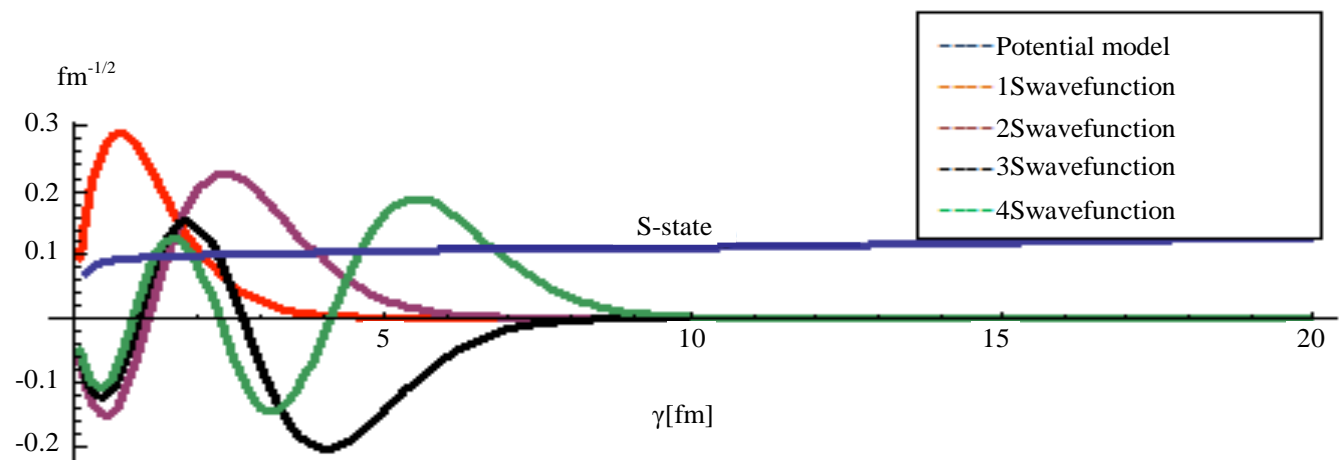

Figure 1. Bottomonium S-states reduced radial wave functions plotted together with used potential.

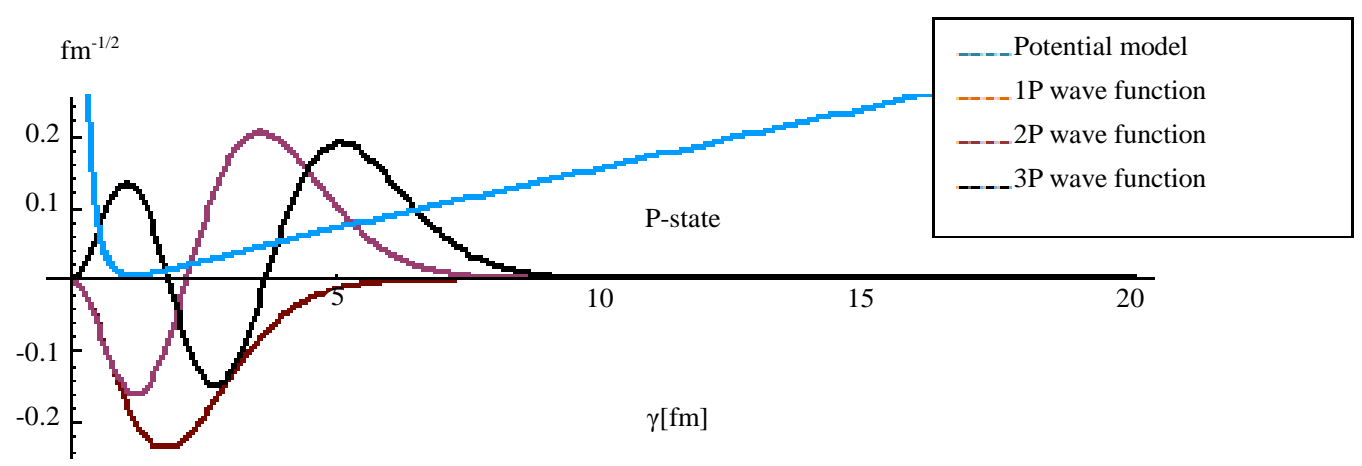

Figure 2. Bottomonium P-states reduced radial wave functions plotted together with used potential. 


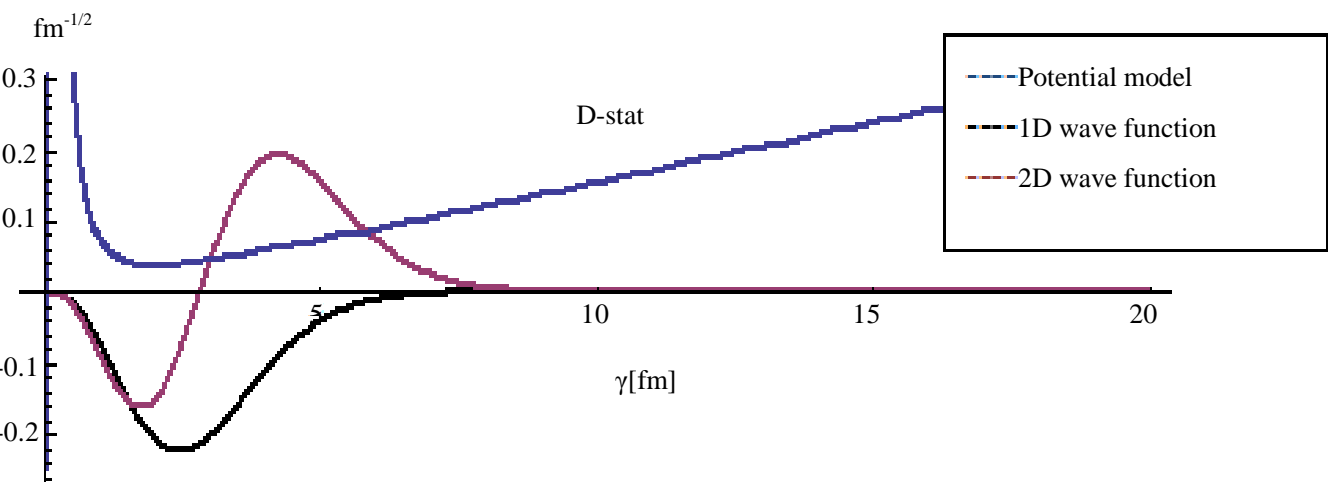

Figure 3. Bottomonium D-state reduced radial wave functions plotted together with used potential.

Table 1. Theoretical masses, the obtained $r_{m s}$ and the values of $\beta$ versus the bottomonium $b \bar{b}$ root mean square radius $r_{m s}$.

\begin{tabular}{|c|c|c|c|}
\hline \multirow{2}{*}{ State } & \multicolumn{3}{|c|}{ Theoretical masses, the obtained $r_{m s}$ and the values of $\beta$} \\
\hline & Theoretical masses in Gev [11] & $r_{m s} f m$ & $\mathrm{~B}$ \\
\hline$\eta_{b}(1 \mathrm{~S}) 1^{1} \mathrm{~S}_{0}$ & 9.393 & 1.01356 & 1.20835 \\
\hline$\eta_{b}(2 \mathrm{~S}) 2^{1} \mathrm{~S}_{0}$ & 9.996 & 2.51449 & 0.74402 \\
\hline $3^{1} \mathrm{~S}_{0}$ & 10.33 & 3.76849 & 0.622321 \\
\hline $4^{1} \mathrm{~S}_{0}$ & 10.596 & 4.8494 & 0.564732 \\
\hline$\Upsilon(1 \mathrm{~S}) 1^{3} \mathrm{~S}_{1}$ & 9.458 & 1.0998 & 1.1136 \\
\hline$\Upsilon(2 S) 2^{3} S_{1}$ & 10.017 & 2.57986 & 0.725166 \\
\hline$\Upsilon(3 S) 3^{3} S_{1}$ & 10.345 & 3.818 & 0.614251 \\
\hline$\Upsilon(4 \mathrm{~S}) 4^{3} \mathrm{~S}_{1}$ & 10.607 & 4.88997 & 0.560047 \\
\hline$\chi_{b 2}(1 \mathrm{P}) 1^{3} \mathrm{P}_{2}$ & 9.936 & 2.11657 & 0.74703 \\
\hline$\chi_{b 2}(2 \mathrm{P}) 2^{3} \mathrm{P}_{2}$ & 10.272 & 3.42884 & 0.61867 \\
\hline$\chi_{b}(3 \mathrm{P}) 3^{3} \mathrm{P}_{\mathrm{J}}$ & 10.539 & 4.54044 & 0.561512 \\
\hline$\chi_{b 1}(1 \mathrm{P}) 1^{3} \mathrm{P}_{1}$ & 9.904 & 1.97379 & 0.801067 \\
\hline$\chi_{b}(2 \mathrm{P}) 2^{3} \mathrm{P}_{1}$ & 10.244 & 3.29457 & 0.643883 \\
\hline$\chi_{b 0}(1 \mathrm{P}) 1^{3} \mathrm{P}_{0}$ & 9.884 & 1.91249 & 0.781072 \\
\hline$\chi_{b 0}(2 \mathrm{P}) 2^{3} \mathrm{P}_{0}$ & 10.234 & 3.26412 & 0.662947 \\
\hline$h_{b}(1 \mathrm{P}) 1^{1} \mathrm{P}_{1}$ & 9.92 & 2.04638 & 0.691082 \\
\hline$h_{b}(2 \mathrm{P}) 2^{1} \mathrm{P}_{1}$ & 10.258 & 3.36292 & 0.630798 \\
\hline$\Upsilon 3(1 \mathrm{D}) 1^{3} \mathrm{D}_{3}$ & 10.166 & 2.83842 & 0.65911 \\
\hline $2^{3} \mathrm{D}_{3}$ & 10.443 & 4.03626 & 0.581035 \\
\hline$\Upsilon 2(1 \mathrm{D}) 1^{3} \mathrm{D}_{2}$ & 10.159 & 2.79207 & 0.67005 \\
\hline $2^{3} \mathrm{D}_{2}$ & 10.437 & 3.99236 & 0.587424 \\
\hline$\Upsilon(1 \mathrm{D}) 1^{3} \mathrm{D}_{1}$ & 10.154 & 2.75709 & 0.678551 \\
\hline $2^{3} \mathrm{D}_{1}$ & 10.432 & 3.95834 & 0.592472 \\
\hline$h_{b 2}(1 \mathrm{D}) 1^{1} \mathrm{D}_{2}$ & 10.162 & 2.80738 & 0.666398 \\
\hline $2^{1} \mathrm{D}_{2}$ & 10.439 & 4.00637 & 0.58537 \\
\hline
\end{tabular}


and the radius for bottomonium and found that, with the exception of the 1S-state, the linear relation is also a good approximation for bottomonium. The relation between mass and radius in case of S-state, P-state and D-state are shown in Figure 4. Moreover, the mass-radius relation for bottomonium $b \bar{b}$ in case of P-state and D-state are shown in Figure 5. It is easily to behold that the S-state seems to be special as it is, in contrast to the other states. It worth noting that, a linear dependence of mass and radius might only be a good guess for states other than the S-state.

\section{Summary and Conclusion}

Bottomium $b \bar{b}$ is predicted by the standard model of particle physics but other models and techniques are required to calculate the particles' properties. The bottomium $b \bar{b}$ meson spectroscopy is studied experimentally

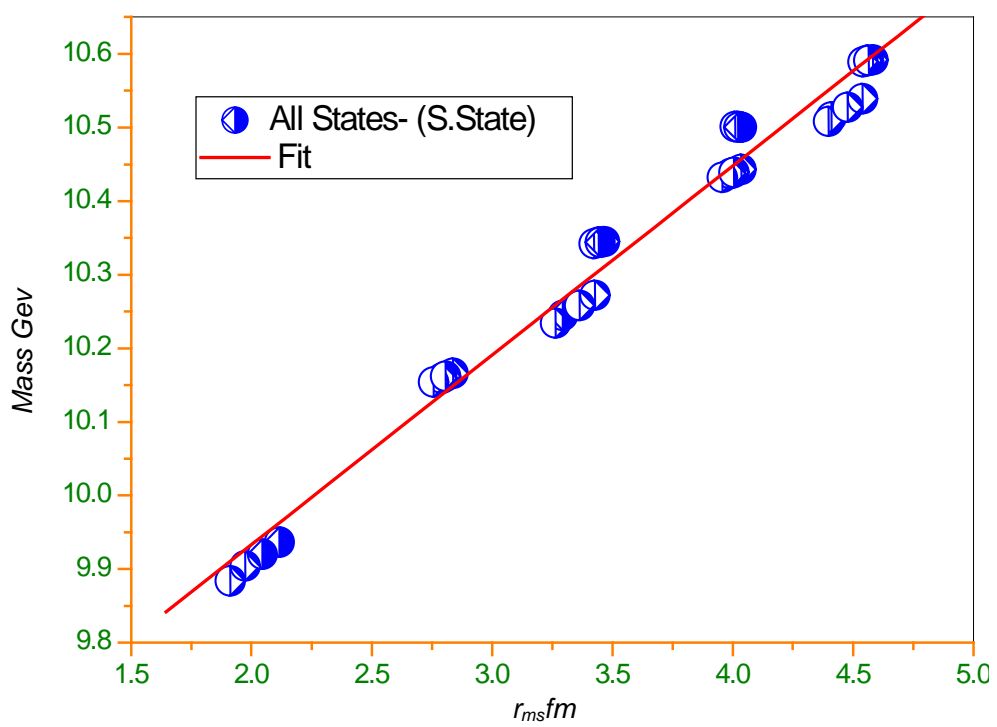

Figure 4. The relation between theoretical spectrum and root mean square $r_{m s}$ of $\mathrm{P}$ and $\mathrm{D}$ bottomonium states.

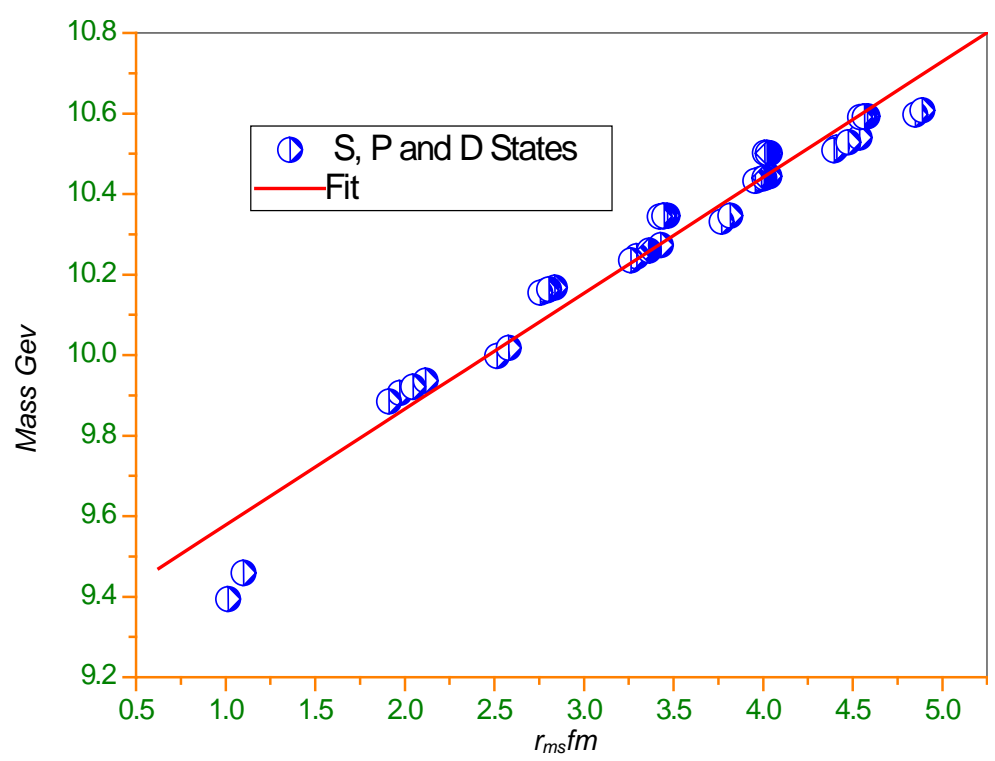

Figure 5. The relation between theoretical spectrum and root mean square $r_{m s}$ of S, P and D bottomonium states. The S-state data has not been included in fitting. 
according to PDG [27]. In this work we use the matrix Numerov's method to obtain the radial wave functions of bottomonium meson to calculate the bottomonium $b \bar{b}$ root mean square $r_{m s}$ and $\beta$ coefficient. As a remarkable result, we can point out that it is recommended to use the obtained values of $r_{m s}$ and $\beta$ coefficient to calculate the decay widths and differential cross sections for bottomonium system. We indicate a high level of accuracy by comparing the results of decay width with available published results of decay width. Moreover, the matrix Numerov's method [11] is tested again to obtain some mesons properties. The obtained results are in good agreement with the published data [28]. Then, the method could be safely used to solve SE. In addition, the relation between theoretical masses we obtained and $r_{m s}$ values have been drawn. As pointed out previously, the fitted straight line reflects perfectly the dependence of mass and radius. Eventually, we may notice that the calculated values of $r_{m s}$ and other parameters are the newer outputs where we didn't find others for comparison. So, we are looking forward to take these data in consideration by other experimental or theoretical researchers.

\section{References}

[1] The ATLAS Collaboration (2011) Physical Review Letters. arXiv:1112.5154v4 [hep-ex]

[2] Buchmuller, W. and Tye, S.H.H. (1981) Physical Review D, 24, 132. http://dx.doi.org/10.1103/PhysRevD.24.132

[3] Moxhay, P. and Rosner, J.L. (1983) Physical Review D, 28, 1132. http://dx.doi.org/10.1103/PhysRevD.28.1132

[4] Godfrey, S. and Isgur, N. (1985) Physical Review D, 32, 189. http://dx.doi.org/10.1103/PhysRevD.32.189

[5] Gupta, S.N., Radford, S.F. and Repko, W.W. (1986) Physical Review D, 34, 201. http://dx.doi.org/10.1103/PhysRevD.34.201

[6] Fulcher, L.P. (1990) Physical Review D, 42, 2337. http://dx.doi.org/10.1103/PhysRevD.42.2337 Fulcher, L.P. (1991) Physical Review D, 44, 2079. http://dx.doi.org/10.1103/PhysRevD.44.2079

[7] Iachello, F., Mukhopadhyay, N.C. and Zhang, L. (1991) Physics Letters B, 256, 295. http://dx.doi.org/10.1016/0370-2693(91)91764-M

[8] Lucha, W., Schoberl, F.F. and Gromes, D. (1991) Physics Reports, 200, 127. http://dx.doi.org/10.1016/0370-1573(91)90001-3

[9] Akbar, N., Masud, B. and Noor, S. (2011) European Physical Journal A, 47, 124. http://dx.doi.org/10.1140/epja/i2011-11124-2

[10] Pillai, M., Goglio, J. and Walker, T.G. (2012) American Journal of Physics, 80, 1017. http://dx.doi.org/10.1119/1.4748813

[11] Yasser, A.M., Hassan, G.S. and Nahool, T.A. (2014) The International Journal of New Horizons in Physics, 2.

[12] Quigg, C. and Rosner, J.L. (1979) Physics Reports, 56, 167-235. http://dx.doi.org/10.1016/0370-1573(79)90095-4

[13] Gershtein, S.S., Kiselev, V.V., Likhoded, A.K. and Tkabladze, A.V. (1995) Physical Review D, 51, Article ID: 3613. http://dx.doi.org/10.1103/PhysRevD.51.3613

[14] Bhaghyesh, Vijaya Kumar, K.B. and Ma, Y.-L. (2012) International Journal of Modern Physics A, 27, Article ID: 1250011.

[15] Simos, T.E. and Aguiar, J.V. (2001) Computers \& Chemistry, 25, 275-281. http://dx.doi.org/10.1016/S0097-8485(00)00101-7

[16] Yang, S. (2007) International Journal of Numerical Analysis and Modeling, 4, 625.

[17] Ixaru, L.G. and Rizea, M. (1980) Computer Physics Communications, 19, 23-27. http://dx.doi.org/10.1016/0010-4655(80)90062-4

[18] Simos, T.E. (2006) Journal of Mathematical Chemistry, 40, 305-318. http://dx.doi.org/10.1007/s10910-006-9170-1

[19] Pedram, P. and Vahabi, M. (2010) American Journal of Physics, 78, 839.

[20] Patel, B. and Vinodkumar, P.C. (2009) Journal of Physics G, 36, Article ID: 035003. http://dx.doi.org/10.1088/0954-3899/36/3/035003

[21] Chaug, C.H., Qiao, C.F. and Wang, J.X. (1998) Physical Review D, 57, Article ID: 4035. http://dx.doi.org/10.1103/PhysRevD.57.4035

[22] Lakhina, O. and Swanson, E.S. (2006) Physical Review D, 74, Article ID: 014012. http://dx.doi.org/10.1103/PhysRevD.74.014012

[23] Barnes, T., Godfrey, S. and Swanson, E.S. (2005) Physical Review D, 72, Article ID: 054026. http://dx.doi.org/10.1103/PhysRevD.72.054026

[24] Aly, A.A. (2012) Heavy Meson Spectra Non-Relativistic Quark Model. M.Sc. Thesis, South Valley University, Qena. 
[25] Zolfagharpour, F. (2008) Nuclear Theory. arXiv:0802.1623 [nucl-th]

[26] Wong, C.-Y. (2004) Physical Review C, 69, Article ID: 055202. arXiv:hep-ph/0311088

[27] Beringer, J., et al., Particle Data Group (2012) Physical Review D, 86, Article ID: 010001. http://dx.doi.org/10.1103/PhysRevD.86.010001

[28] Gupta, P. and Mehrotra, I. (2010) Nuclear Physics, 55, 548.

[29] Patel, B. and Vinodkumar, P.C. (2009) Journal of Physics G, 36, Article ID: 035003. http://dx.doi.org/10.1088/0954-3899/36/3/035003

[30] Chaug, C.H., Qiao, C.F. and Wang, J.X. (1998) Physical Review D, 57, Article ID: 4035. http://dx.doi.org/10.1103/PhysRevD.57.4035 
Scientific Research Publishing (SCIRP) is one of the largest Open Access journal publishers. It is currently publishing more than 200 open access, online, peer-reviewed journals covering a wide range of academic disciplines. SCIRP serves the worldwide academic communities and contributes to the progress and application of science with its publication.

Other selected journals from SCIRP are listed as below. Submit your manuscript to us via either submit@scirp.org or Online Submission Portal.
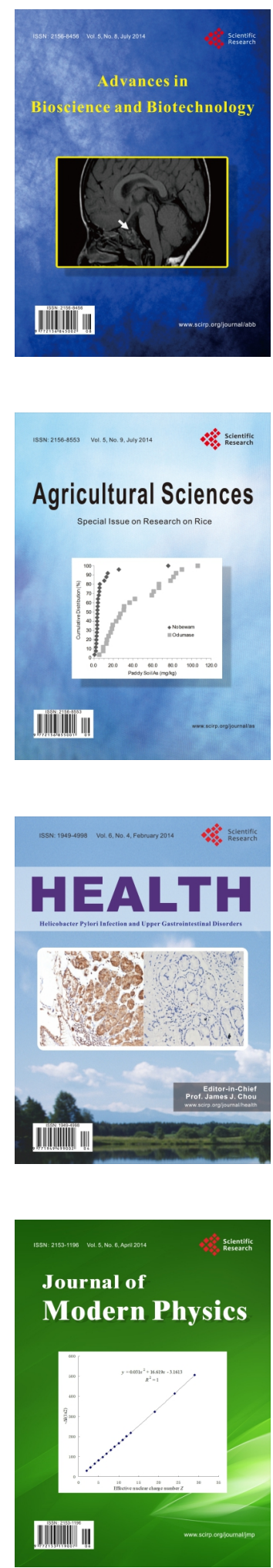
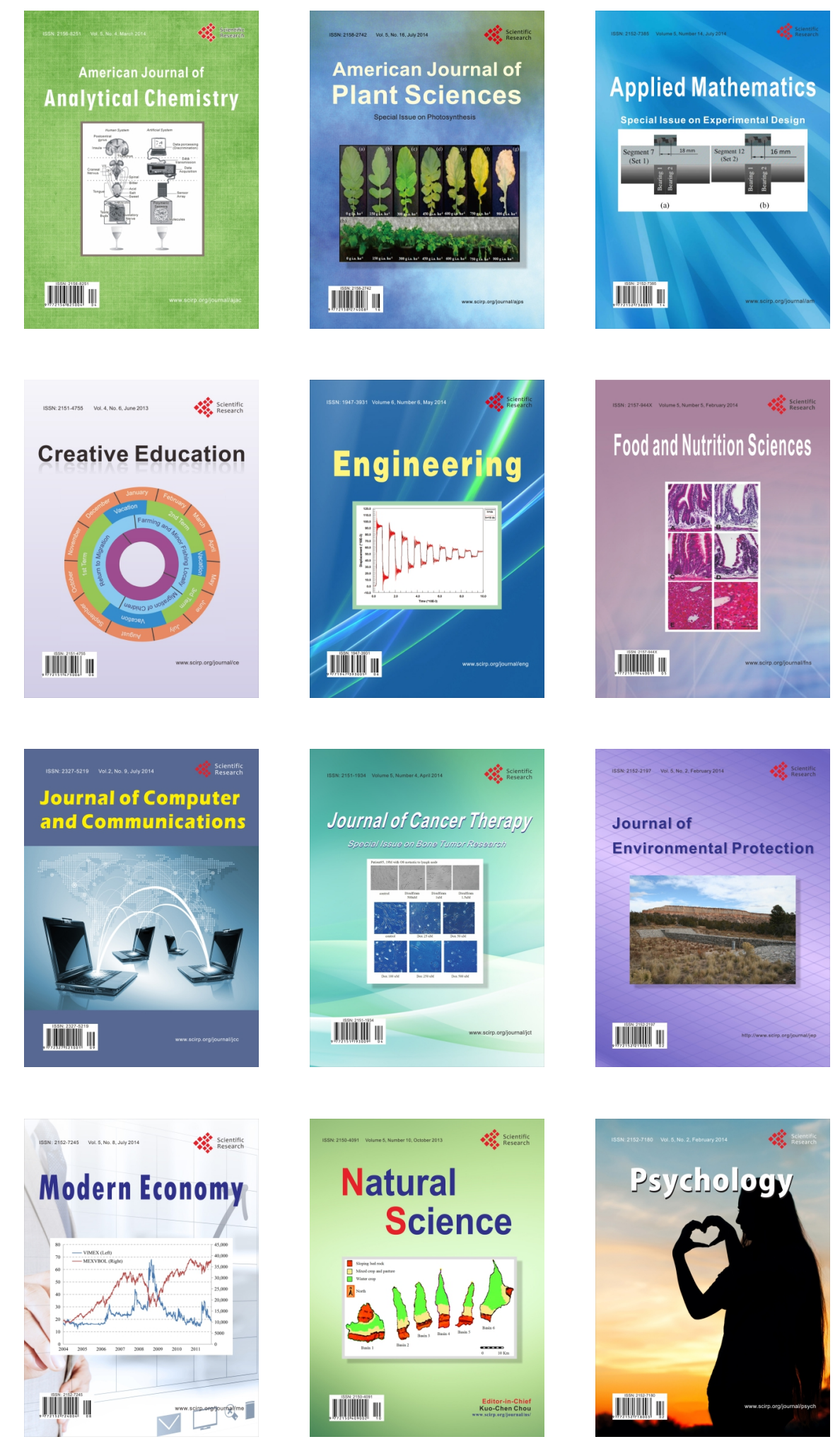